\title{
Quinones as synthon for construction of heterocyclic systems with anticipated bioactivity
}

\author{
Alaa E. Hassanien \\ Chemistry Department, Faculty of Science, Mansoura University, Mansoura, Egypt \\ Basic Science Department, Higher Future institute of Technology and Engineering, Mansoura, Egypt
}

Background: Lawsone (2-hydroxynaphthalene-1,4-dione) is a natural product that shows significant biological activity. The National Cancer Institute (NIH, United State) disclosed the lawsone skeleton that contains the quinone moiety as a precursor for clinically cytotoxic activity. Aim: Lawsone has interesting pharmacological performance, particularly as antitumor, antimicrobial, anti-parasite (Trypanosoma cruzi), and Leishmanicidal activity, anti-lung cancer. Materials and Methods: Three different approaches were taken in the synthetic approach. The first approach was based on the synthesis of naphthoquinone annulation that was synthesized from the reaction of 2 with different binucleophilic reagents via Michael addition followed by intramolecular condensation. The second approach was based on the synthesis of new $\alpha$-lapachone analogs during the cyclo-condensation reaction of 1 with $\alpha, \beta$-unsaturated ketones. The third approach was based on the synthesis of novel spiro lawsone-ring skeletons, which focuses on the synthesis of spiro lawsone ring skeletons by ClaisenSchmidt condensation of 1 with some selected advanced reagents. Results: Pharmacological studies of the synthetic compounds provided an indication of biological activities, including effects against hepatocellular carcinoma (HePG-7) and mammary gland breast cancer (MCF-7). The molecular structures of some molecules were examined by using DFT calculations. Conclusion: The synthetic compounds possess significant anti-tumor capabilities.

Keywords: Alpha Lapachone; Anti-Oxidant; Anti-Tumor; Geometrical optimization; Lawsone

Editor-in-Chief: Prof. M.L. Salem, PhD - Article DOI: 10.21608/JCBR.2021.60637.1155 